\title{
Specific Disulfide Bond Formation Directed by Peptide Nucleic Acids
}

\author{
Lin $\mathrm{Cai}^{a}$ and Wen $\mathrm{Hou}^{\circledR *, b, c, d}$ \\ ${ }^{a}$ Gerontology Department, Tianjin First Central Hospital, 300192 Tianjin, China \\ ${ }^{b}$ Key Laboratory for Critical Care Medicine of the Ministry of Health, \\ Tianjin First Central Hospital, 300192 Tianjin, China \\ ${ }^{c}$ State Key Laboratory of Medicinal Chemical Biology, Nankai University, 300071 Tianjin, China \\ ${ }^{d}$ Key Laboratory of Transplantation of Chinese Academy of Medical Sciences, \\ Tianjin First Central Hospital, 300192 Tianjin, China
}

\begin{abstract}
In this study, we successfully demonstrated a strategy for specific disulfide bond formation by hybridization of two complementary peptide nucleic acid strands. Peptides were linked to the pair of complementary peptide nucleic acids by native chemical ligation, respectively, and base pairing drove the two peptides into close proximity for interchain disulfide bond formation. Since peptide nucleic acids directed system is easier to manipulate than deoxyribonucleic acid system, this system will be more useful and have a broader application scope in the construction of complex biomolecular structures.
\end{abstract}

Keywords: peptide nucleic acid, disulfide bond, heterodimerization

\section{Introduction}

Peptide nucleic acid (PNA) is an oligonucleotide mimic in which the (deoxy)ribose-phosphate backbone has been replaced by an $N$-(2-aminoethyl)glycine unit and the nucleobases (adenyl (A), cytosyl (C), guanidyl (G), and thymidyl (T)) are attached to the backbone through methylenecarbonyl linkages (Figure 1). ${ }^{1}$ The amine terminus of PNA is analogous to the 5'-hydroxyl of deoxyribonucleic acid (DNA). PNA has both the characteristics of peptide and nucleic acid. The PNA residues are linked by amide bond like peptide and PNA has the hybridization properties of nucleic acids originally detailed by Watson and Crick in 1953. ${ }^{2}$ Since the backbone of PNA does not contain charged phosphate groups, the complementary PNA/PNA strands or PNA/DNA or PNA/RNA (ribonucleic acid) strands are more stable and have higher melting temperature $(\mathrm{Tm})$ than the<smiles>[B]CC(=O)N(CCNC)CC(=O)NCCN(CC(C)=O)C(=O)CBr</smiles>

Figure 1. Structure of PNA.

*e-mail: houwen_fch@126.com complementary DNA/DNA strands. This property makes it not only a potential antisense and antigene drug candidate, but also useful in other applications, such as solid phase hybridization, nucleic acid capture and bioconjugation. ${ }^{3}$ Moreover, PNA has been found to hybridize to PNA and DNA (or RNA) in both the parallel and antiparallel modes, though antiparallel is preferred.

PNA is completely acid stable even under HF conditions. PNA is also stable in the condition of weak bases, such as piperidine. Therefore, PNA can be synthesized either by standard tert-butyloxycarbonyl (Boc) or 9-fluorenylmethoxycarbonyl (Fmoc) solid phase peptide synthesis (SPPS).

Our strategies are utilizing the hybridization properties of two complementary strands of PNA to bring together two peptides or proteins attached to each strand to approach some biological or chemical activities, such as directed disulfide bonds formation. Actually, this idea was originally inspired by the DNA-directed assembly of enzyme complexes, which was reviewed below.

In biological systems, multi-enzyme complexes have mechanistic advantage that they avoid the multistep catalytic transformation of a substrate and accelerate the reaction rate which was limited by diffusional transport. An early demonstration of bienzymic complexes which 
contains nicotinamide adenine dinucleotide (NADH): flavin mononucleotide (FMN) oxidoreductase and luciferase by DNA-directed assembly was developed. ${ }^{4}$ The conjugation of DNA and enzyme was based on the high affinity of biotin-streptavidin coupling system. Later on, the bienzymatic complex of glucose oxidase (GOx) and horseradish peroxidase (HRP) bound in direct proximity on the DNA carrier strand showed the best bienzymatic reactivity of those complexes. ${ }^{5}$ Willner and co-workers ${ }^{6,7}$ have also investigated the effects of DNA assembly on the dienzymic system of GOx and HRP. Moreover, multistep synthesis of biomolecules can be achieved by using DNA-templation. The chemical groups attached to the DNA adapters can be approached in close proximity by means of DNA hybridization, thus the effective molarity of reactive species was increased and the reaction was accelerated. ${ }^{8}$ After that, a strategy for the synthesis of ordered functional oligomers by means of sequential DNAtemplated reactions, using a strand displacement system, was developed. ${ }^{9}$ There were also many studies on the multistep DNA-templated synthesis using a linear template or DNA multibranched junction to encode the sequence of the desired product. ${ }^{10-12}$

From the research reviewed above, DNA can be used as a carrier to bring two different chemical groups in close proximity to achieve more than two biological or chemical activities. We thought PNA was a better substitute with the following reasons. Firstly, PNA synthesis is mostly like peptide synthesis using commercial available PNA monomers by the widely used Boc or Fmoc SPPS. Secondly, with its peptide-like features, PNA can be ligated to another peptide by chemical ligation methods ${ }^{13,14}$ in place of the complicated biotin-streptavidin coupling system for conjugation between the DNA and protein. Thirdly, the complementary PNA/PNA can be bound by parallel mode, which can simplify the procedures to prepare PNA-peptide conjugates. Finally, PNA is not recognized by nucleases and proteases, and can be stored longer without degradation as compared to DNA or RNA. Above all, we chose PNA as a carrier for peptide/protein assembling.

We report a system for peptide heterodimerization through PNA-templated disulfide bond formation (Figure 2). In this system, a pair of complementary PNAs is respectively linked to two peptides and the PNA base pairing brings the two peptides into close proximity for interchain disulfide bond formation. The results show that this reaction system is highly selective with the desired heterodimer formed exclusively. Since PNA synthesis is fully compatible with peptide synthesis, this method has an advantage over and can be more useful than the DNA-templated reaction systems.

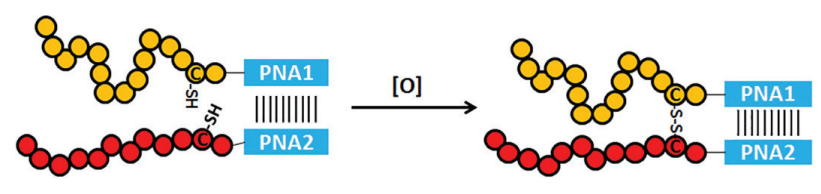

Figure 2. Specific disulfide bond formation directed by PNA hybridization.

\section{Experimental}

Materials

All PNA monomers were purchased from ASM Research Chemicals (Hannover, Germany). The rink amide PEGA resin, 4-methylbenzhydrylamine (MBHA) resin, all the amino acids and coupling reagents were purchased from GL Biochem (Shanghai, China) and Novabiochem (Darmstadt, Germany). All other chemical reagents were purchased from commercial suppliers.

\section{Methods}

\section{Synthesis of PNA}

Rink amide PEGA resin was swelled in dimethylformamide/dichloromethane (DMF/DCM 5/1) for $2 \mathrm{~h}$. The Fmoc group was deprotected by $20 \%$ piperidine in DMF twice $(2 \min \times 1,20 \min \times 1)$. After washing the resin with DMF $(3 \times)$ and DCM $(3 \times)$, Fmoc-Gly-OH was coupled onto the resin by Fmoc-SPPS method. The procedure of coupling the monomers was almost the same as Fmoc-SPPS with a slight modification. The Fmoc group was deprotected by $20 \%$ piperidine in DMF twice in shorter time $(1 \min \times 1,3 \min \times 1)$. The monomer and benzotriazol1-yl-oxytripyrrolidinophosphonium hexafluorophosphate (PyBOP) were used in twofold excess of resin other than fourfold in standard Fmoc-SPPS, and the coupling time was prolonged to 4-6 h. Ninhydrin test was used throughout the synthesis to monitor the coupling and deprotection steps. The Fmoc deprotection and monomer coupling cycles were continued until the last residue. After the final Fmoc deprotection, the resin was washed thoroughly with DMF/ DCM and dried in vacuo. The resin was cleaved by $95 \%$ trifluoroacetic acid (TFA), $2 \% \mathrm{H}_{2} \mathrm{O}, 1.5 \%$ triisopropylsilane (Tis), $1.5 \%$ 2-mercaptoethanal in volume for $1 \mathrm{~h}$. The PNA was precipitated by ethyl ether, and then lyophilized and purified by high performance liquid chromatography (HPLC).

The sequence of PNA1 is Cys-Ala-GTAGATCACT-Gly. The sequence of PNA2 is Cys-Ala-CATCTAGTGA-Gly. The side chain of Cys was protected by trityl (Trt), and the free amine groups on the bases of the PNA monomers (A, C, G) were protected by Boc. 


\section{Synthesis of peptide thioesters}

Peptide GCN thioester and peptide Max thioester were synthesized by manual SPPS Boc chemistry using MBHA resin. First of all, the thio-functionalized MBHA resin was prepared by coupling Trt- $\mathrm{SCH}_{2} \mathrm{CH}_{2} \mathrm{COOH}$ directly to the MBHA resin. Trityl group was removed by $5 \%$ TFA three times, and detritylation was confirmed by Ellman test.

Then peptides were assembled on the thiol-derived resin using typical SPPS protocols. All the protected amino acids were used in 4 equiv. of the resin, and preactivated by 4 equiv. of PyBop and 10 equiv. of $\mathrm{N}, \mathrm{N}$-diisopropylethylamine (DIEA). After one hour coupling of the amino acid, the resin was washed by DCM $(3 \times), \operatorname{DMF}(3 \times)$ and DCM (3x). Boc was removed by $30 \%$ TFA in DCM two times, for 1 and 20 min, respectively. Then the resin was washed by DCM (3x), DMF ( $3 x$ ) and $\operatorname{DCM}(3 \times)$. Then the coupling and deprotection procedure were followed again as described before.

The sequence of GCN is H-LKDPAALKRARNTEAA RRSRARKLQRA-SCH${ }_{2} \mathrm{CH}_{2} \mathrm{CONH}_{2}$. The following side chain protecting groups were used for peptide $\mathrm{GCN}$ : benzyl (Bzl) for threonine (Thr), cyclohexyl ester (OcHx) for Asp and Glu, Trt for Asn and Gln, toluenesulfyl (Tos) for Arg, 2-chlorobenzyloxycarbonyl (2-Cl-z) for Lys, Bzl for Ser. The peptide resin was cleaved by HF procedure. The peptides were purified by HPLC.

The sequence of Max is H-ADKRAHHNALERK RRDHA-SCH $\mathrm{CH}_{2} \mathrm{CONH}_{2}$. The following side chain protecting groups were used for peptide Max-18: OcHx for Asp and Glu, Trt for Asn, Tos for Arg and His, and 2-Cl-z for Lys. The peptide resin was cleaved by HF procedure. The peptide was purified by HPLC.

\section{Native chemical ligation of the GCN peptide thioester with PNA1}

The above peptide thioester (final concentration $10 \mathrm{mM}$ ) and PNA (final concentration $3 \mathrm{mM}$ ) were added to $20 \mathrm{mM}$ tris(2-carboxyethyl)phosphine (TCEP), $6 \mathrm{M}$ guanidine.hydrogen chloride $(\mathrm{Gdn} . \mathrm{HCl}), 200 \mathrm{mM}$ 4-(2-hydroxyethyl)-1-piperazineethanesulfonic acid (Hepes) pH 8 buffer. Then $2 \%(\mathrm{~m} / \mathrm{v})$ thiophenol and $2 \%$ $(\mathrm{m} / \mathrm{v})$ 2-mercaptoethanesulfonate sodium (MESNa) were added. $\mathrm{pH}$ was adjusted to 8.5 . The reaction was incubated at room temperature for $6 \mathrm{~h}$. The ligation product GCNPNA1 was purified by HPLC.

\section{Ligation of PNA2 with Max thioester}

The condition of the native chemical ligation was all the same as above. The ligation product Max-18-PNA2 was purified by HPLC.

\section{Hybridization}

The same concentration of GCN-PNA1 and Max-PNA2 were hybridized. Two strands of ligation products were dissolved in a buffer solution containing $100 \mathrm{mM} \mathrm{NaCl}$, $0.1 \mathrm{mM}$ ethylenediaminetetraacetic acid (EDTA), and $20 \mathrm{mM}$ phosphate buffer ( $\mathrm{pH}$ 7.0). The whole solution was heated at $90{ }^{\circ} \mathrm{C}$ for $2 \mathrm{~min}$ in a water bath, and cooled to room temperature gradually. Thermal denaturation studies (Tm) were run on a Cary 300 Bio UV-Visible spectrophotometer. The concentration of double strand PNA (dsPNA) used for Tm studies was $2 \mu \mathrm{M}$. The sample was first heated to $90^{\circ} \mathrm{C}$ for $5 \mathrm{~min}$, followed by gradually cooling to room temperature. UV-absorption was monitored at $260 \mathrm{~nm}$ from 80 to $20^{\circ} \mathrm{C}$ and 20 to $80{ }^{\circ} \mathrm{C}$ at the rate of $0.5^{\circ} \mathrm{C}$ per minute. All values are accurate to $1^{\circ} \mathrm{C}$.

\section{Oxidation}

Different concentrations of double strands of GCN-PNA1 and Max-PNA2 at $0.5,1,5,10$ and $20 \mu \mathrm{M}$, respectively, were exposed to air overnight. After desalination by $\mathrm{C} 18$ ZipTip, the oxidation products were detected by matrixassisted laser desorption/ionization time-of-flight mass spectrometry (MALDI-TOF MS).

\section{Results and Discussion}

\section{Synthesis and purification of PNA1 and PNA2}

PNA was successfully synthesized by Fmoc SPPS protocol with minor modifications. Fmoc was deprotected by $20 \%$ piperidine in DMA twice in shorter time $(1 \min \times 1,3 \min \times 1)$ for two reasons. One is to avoid the potential side reactions involving the amine terminus and the acyl moieties of the nucleobase tether or the interunit linkage (Figure 3). The other reason is the less hindrance in the vicinity of amine in PNA monomers comparing to normal amino acids. Therefore, the deprotection reaction is more rapid.

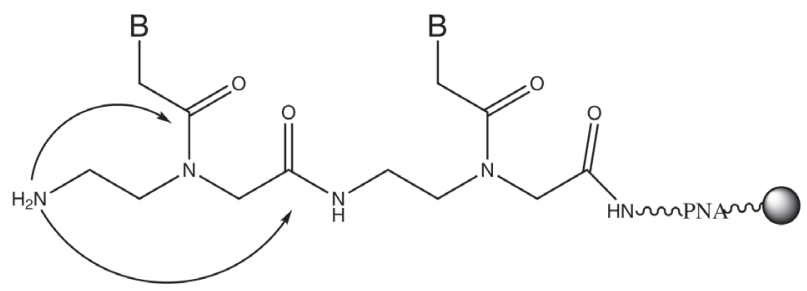

Figure 3. Potential transacylation pathways.

A Cys-Ala dipeptide was added to the N-terminus of the two PNAs to facilitate the attachment of peptide thioester via chemical ligation. The PNA was cleaved 
by TFA under typical conditions. After purification by HPLC, purified PNA1 and PNA2 were obtained (Figures 4 and 5).

\section{Synthesis and purification of GCN thioester}

GCN thioester, with the sequence of H-LKDPAALKRA RNTEAARRSRARKLQRA-SCH${ }_{2} \mathrm{CH}_{2} \mathrm{CONH}_{2}$, was synthesized on MBHA resin by standard Boc SPPS. The thioester bond was directly generated on the MBHA resin by using S-trityl mercaptopropionic acid. The amino acids were assembled onto the mercaptopropionyl MBHA resin

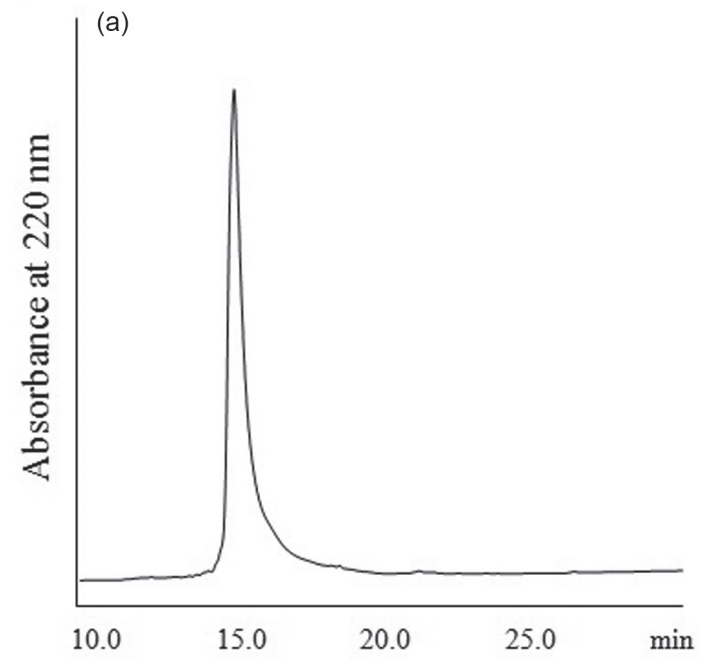

following standard SPPS protocols. After HF deprotection and cleavage, the peptide thioester was released from the resin. The peptide thioester was purified by RP-HPLC and the molecular weight was determined by ESI-MS. The analytical reversed-phase liquid-cromatography (RP-HPLC) and MALDI-TOF-MS of GCN thioester are shown below (Figure 6).

\section{Ligation of PNA1 and GCN thioester}

The reaction of native chemical ligation between PNA1 and GCN thioester was checked by analytical

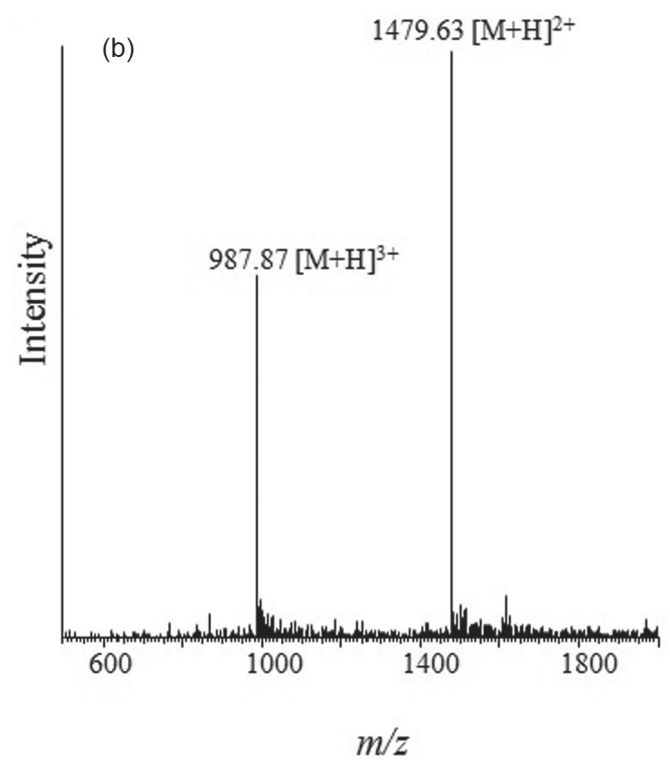

Figure 4. Characterization of PNA1. (a) C18 analytical HPLC profile of PNA1. HPLC condition: 0 to $60 \%$ of buffer B in buffer A in 30 min (buffer A was distilled deionized water containing $0.05 \%$ TFA and buffer B was $90 \%$ acetonitrile in distilled water containing $0.05 \%$ TFA). (b) Mass spectrum of this PNA determined by electrospray ionization (ESI-MS). [M + 2H $]^{2+}$ found: 1479.63, molecular weight (MW) calcd. 2957.1.

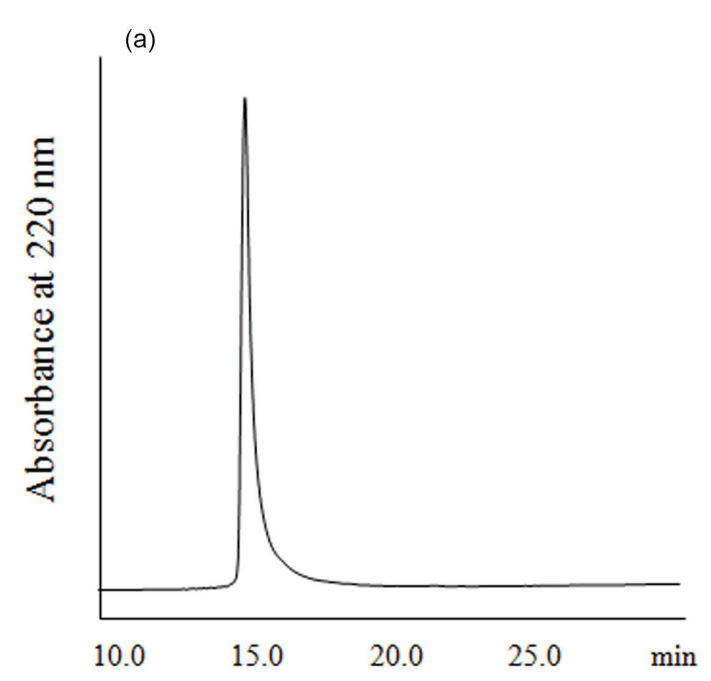

Retention time

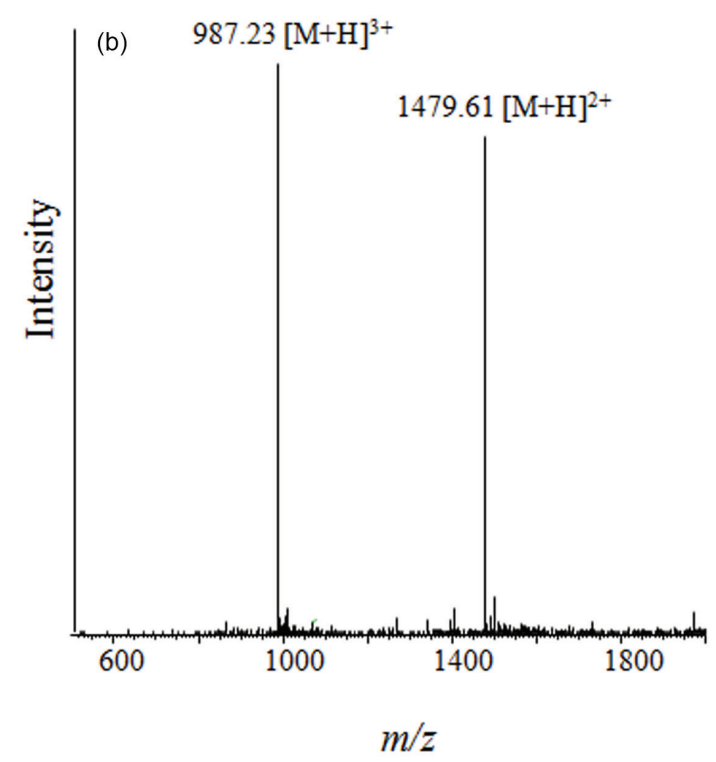

Figure 5. Characterization of PNA2. (a) C18 analytical HPLC profile of PNA2. HPLC condition: 0 to $60 \%$ of buffer B in buffer A in 30 min. (b) Mass spectrum of this PNA determined by ESI-MS. $[\mathrm{M}+2 \mathrm{H}]^{2+}$ found: 1479.61, MW calcd. 2957.2. 

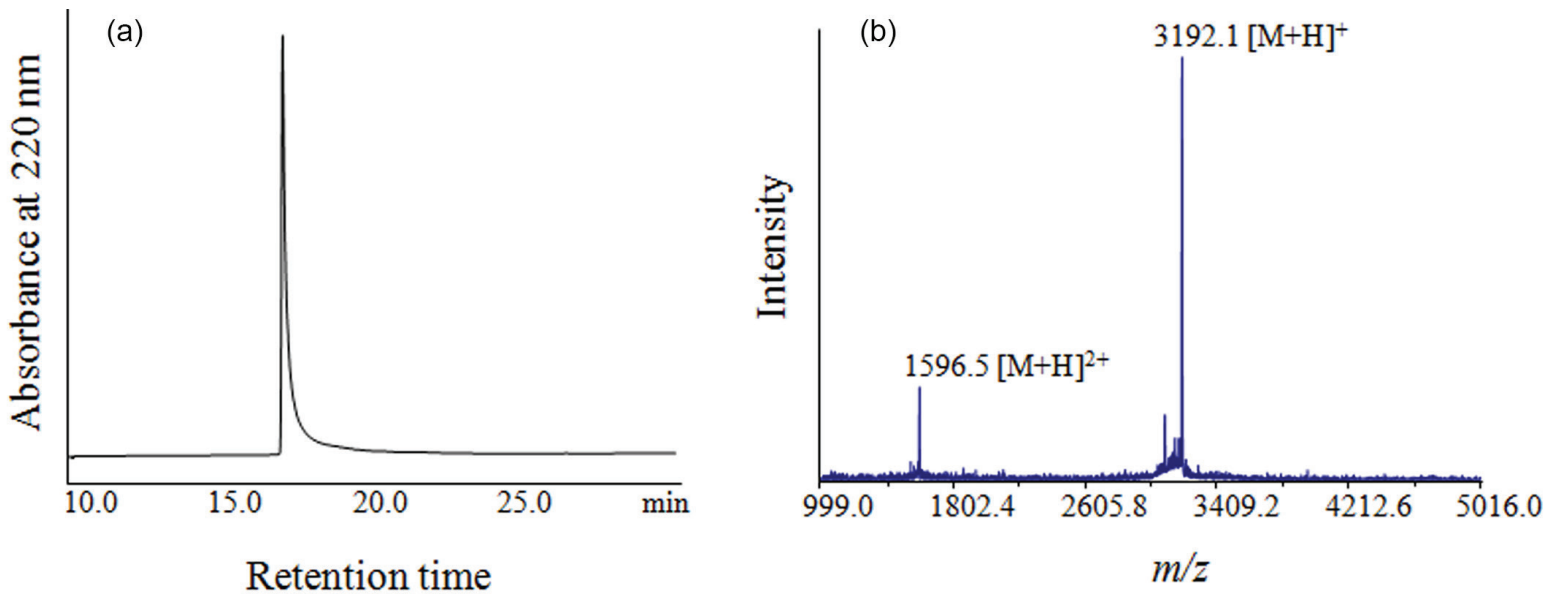

Figure 6. Characterization of peptide GCN thioester. (a) C18 analytical HPLC profile of peptide GCN thioester. HPLC condition: 0 to $60 \%$ of buffer B in buffer A in 30 min. (b) Mass spectrum of this peptide determined by MALDI-MS. [M + H] found: 3192.1, MW calcd. 3191.2.

RP-HPLC and the reaction was completed after $6 \mathrm{~h}$. In Figure 7, peak 1 (PNA) and peak 2 (GCN thioester) are the substrates in control. Even though the absorbance of peak 1 is much higher than peak 2, GCN thioester was 3 times in excess. The high absorbance of PNA was due to many purines and pyrimidines in the structure. Peak 3 was the ligation product, and peak 4 was the GCN MES thioester remained, since MESNa was used as the additive in this ligation.

The MALDI-TOF mass spectrum of ligation product is in Figure 8.

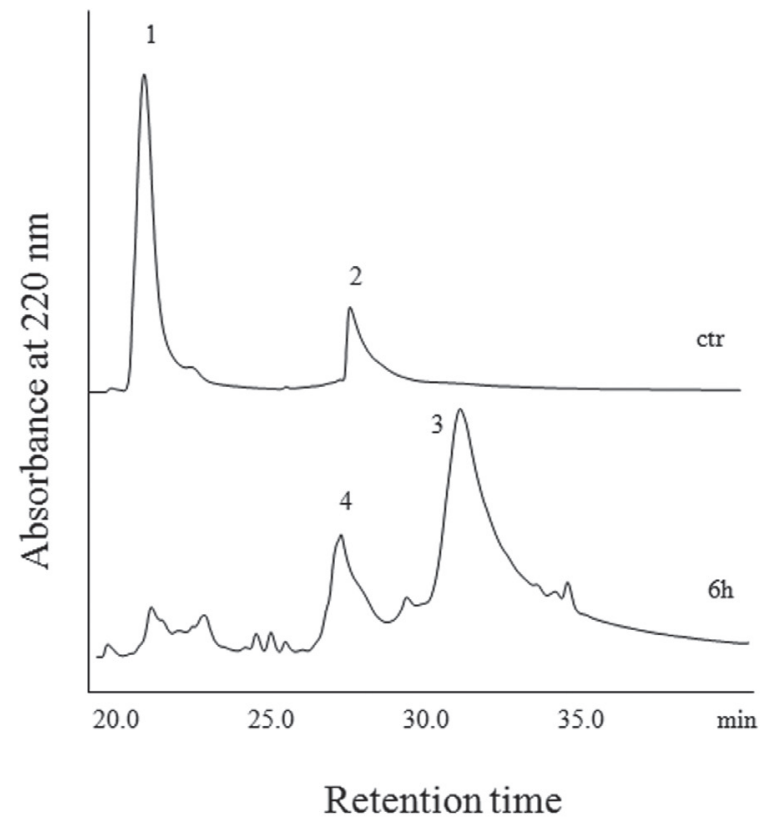

Figure 7. $\mathrm{C} 18$ analytical HPLC analysis of the ligation reaction. HPLC conditions: 0 to $15 \%$ of buffer B in buffer A in $15 \mathrm{~min}$ and then 15 to $25 \%$ of buffer B in buffer A in 20 min. Peak 1 is PNA1, peak 2 is GCN thioester, peak 3 is the ligation product and peak 4 is the MESNa exchange product of GCN thioester.

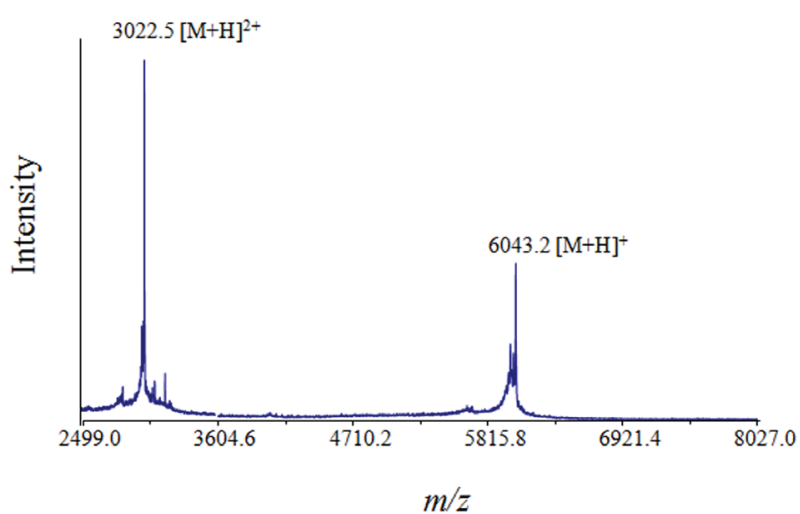

Figure 8. Mass spectrum of the ligation product GCN-PNA1 determined by MALDI-MS. [M + H] $]^{+}$found: 6043.2 , MW calcd. 6042.2.

Synthesis and purification of Max thioester

Max thioester, with the sequence of H-ADKRAHHNA LERKRRDHA-SCH $\mathrm{CH}_{2} \mathrm{CONH}_{2}$, was synthesized on MBHA resin by standard Boc SPPS as described above. The MALDI-MS and analytical RP-HPLC spectra of Max18 are as below (Figure 9).

Ligation of PNA2 and Max thioester

The PNA2 was ligated to Max thioester also by native chemical ligation. The reaction between PNA2 and Max was checked by analytical RP-HPLC (Figure 10) and the reaction was completed after $6 \mathrm{~h}$.

The MALDI-mass spectrum of ligation product is in Figure 11.

Hybridization

GCN-PNA1 and Max-PNA2 were dissolved in a buffer $(\mathrm{pH} 7)$ at equal concentration. Then, the sample was first 
(a)

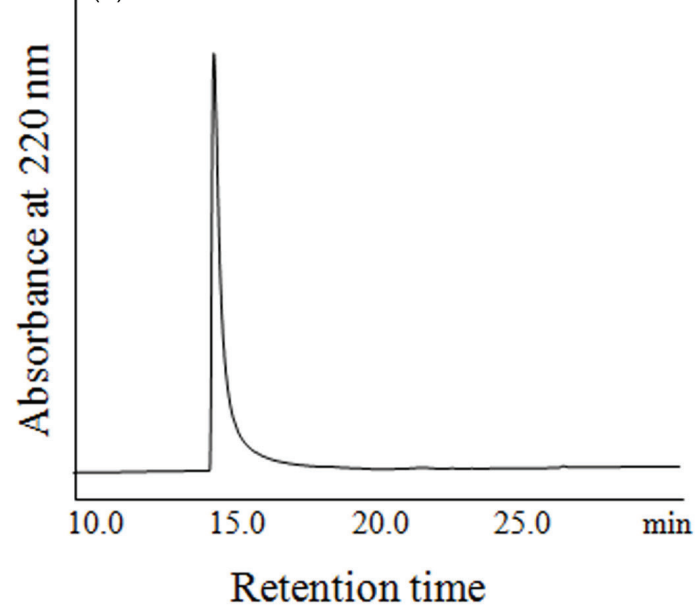

(b) $2268.31[\mathrm{M}+\mathrm{H}]^{+}$

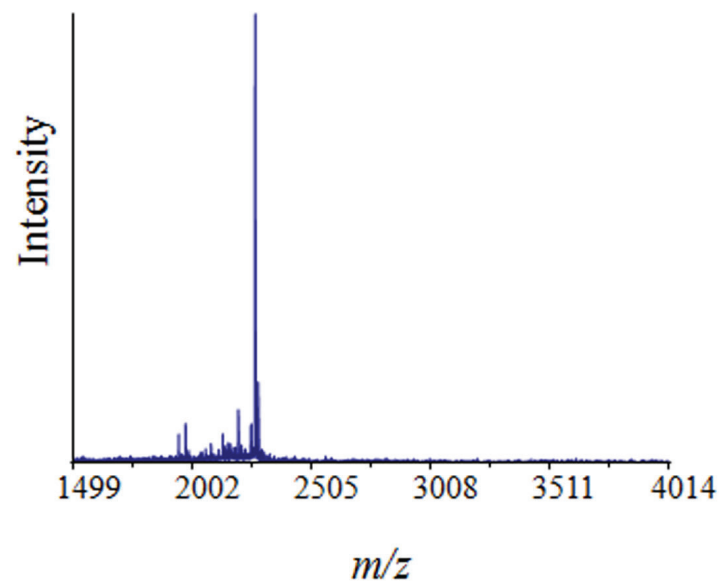

Figure 9. Characterization of peptide Max thioester. (a) C18 analytical HPLC profile of peptide Max thioester. HPLC condition: 0 to $60 \%$ of buffer B in buffer A in 30 min. (b) Mass spectrum of this PNA determined by MALDI-MS. [M + H] $]^{+}$found: 2268.31, MW calcd. 2267.2.

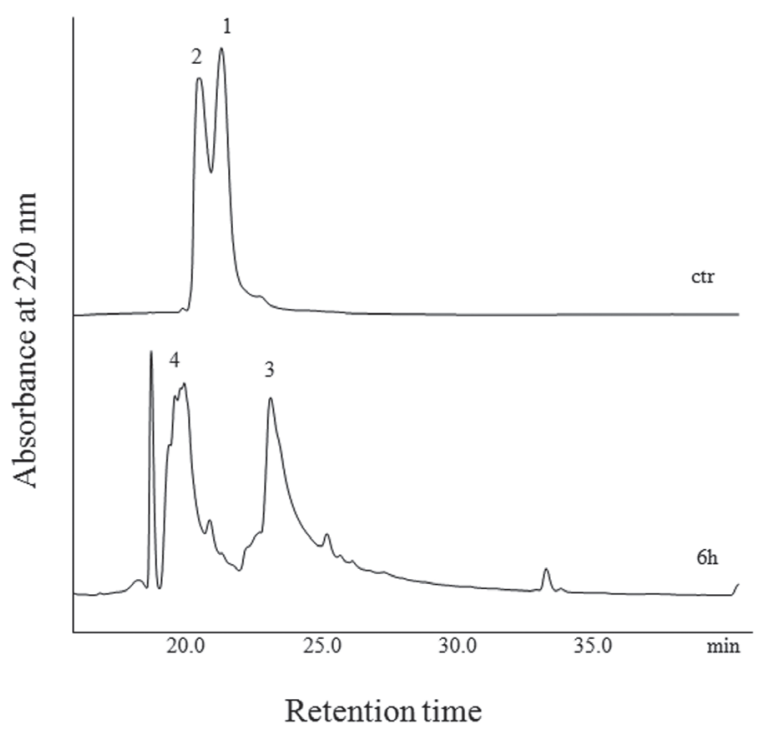

Figure 10. C18 analytical HPLC analysis of the ligation reaction. HPLC conditions: 0 to $15 \%$ of buffer B in buffer A in 15 min and then 15 to $25 \%$ of buffer B in buffer A in 20 min. Peak 1 is PNA1, peak 2 is Max thioester, peak 3 is the ligation product and peak 4 is the MESNa exchange of Max thioester.

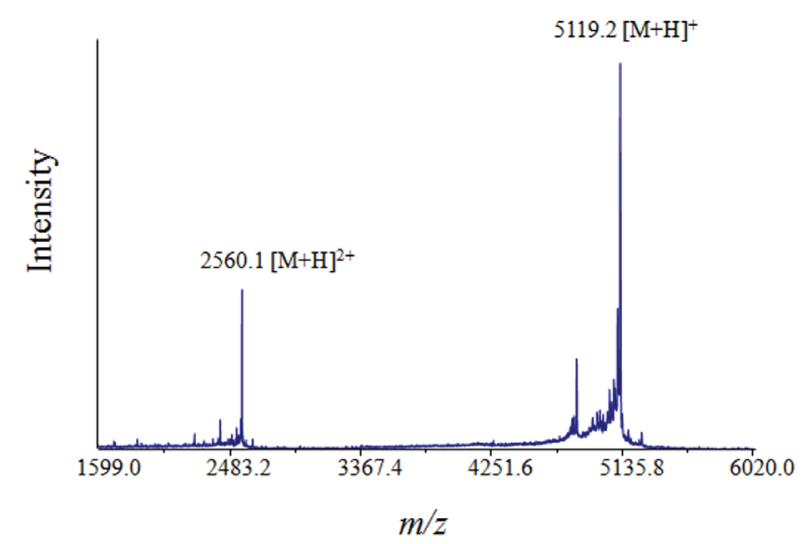

Figure 11. Mass spectrum of this peptide determined by MALDI-MS. $[\mathrm{M}+\mathrm{H}]^{+}$found: 5119.2, MW calcd. 5118.2.

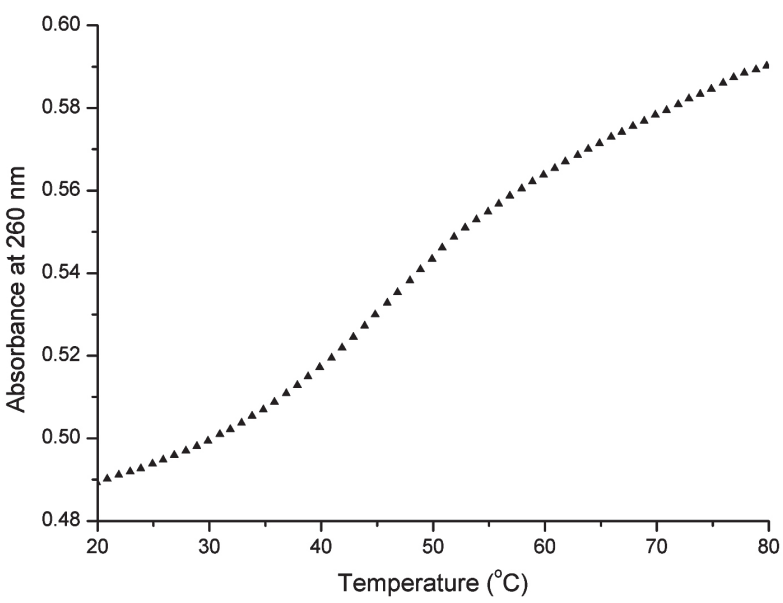

Figure 12. Melting curve spectrum of dsPNA.

heated to $90^{\circ} \mathrm{C}$ for $3 \mathrm{~min}$, followed by gradually cooling to room temperature. After hybridization, we took $2 \mu \mathrm{M}$ GCN-PNA1 and Max-PNA2 in $100 \mu \mathrm{L}$ volume to do the melting curve analysis (Figure 12). A nice $\mathrm{S}$ curve showed that the two strands of PNA hybridized well to form a duplex.

\section{Detection of unsymmetric disulfide bond formation}

After hybridization of the two PNA strands and oxidation, MALDI-MS was used to detect formation of heterodimers of GCN-PNA1 and Max-PNA2 joined together through a disulfide bridge. If the disulfide bond forms between two GCN4-PNA1 molecules, the mass of the product will be the double mass of GCN-PNA1 minus 2 . The same rule applies to the homodimer of Max-PNA2 molecules. However, if the dimmer is a heterodimer of GCN-PNA1 and Max-PNA2, the MW of the covalently linked heterodimer will be the sum of MW of the two 
minus 2. These different molecular masses can be easily distinguished by MALDI-MS.

Different concentrations of double strands of GCN-PNA1 and Max-PNA2 were oxidized overnight in the air at $0.5,1,5,10$ and $20 \mu \mathrm{M}$ in totally $50 \mu \mathrm{L}$ reaction solution. After desalting, $10 \mu \mathrm{L}$ aliquot of each reaction mixture was loaded on a metal plate for MALDI-MS analysis (Figure 13). Simple dsPNA complex is not stable enough to be detected on MALDI-TOF MS, because the binding between the two strands are non-covalent and would be dissociated during the laser desorption ionization process in the MALDI-TOF machine (Figure 13a). From the results, only one form of disulfide bond was detected, that is the one formed between GCN-PNA1 and Max-PNA2. There were no other disulfide bonds formed

(a)

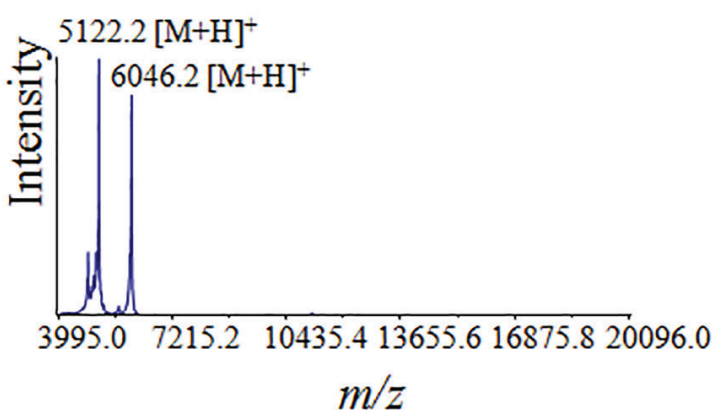

(c)

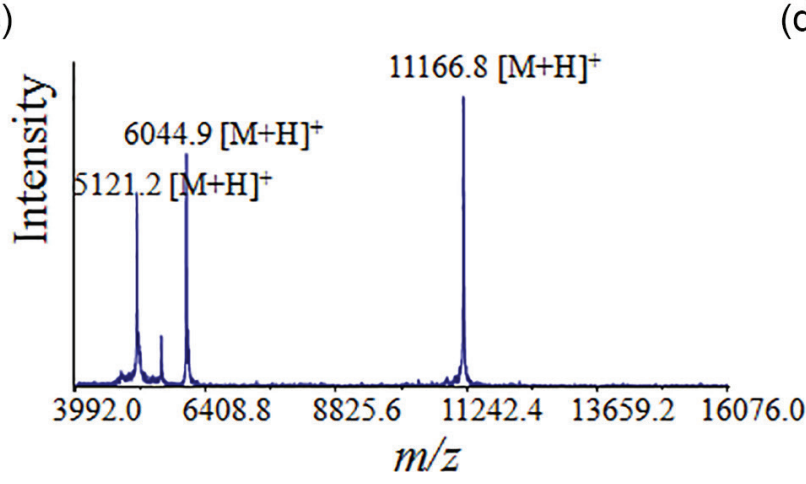

(b)

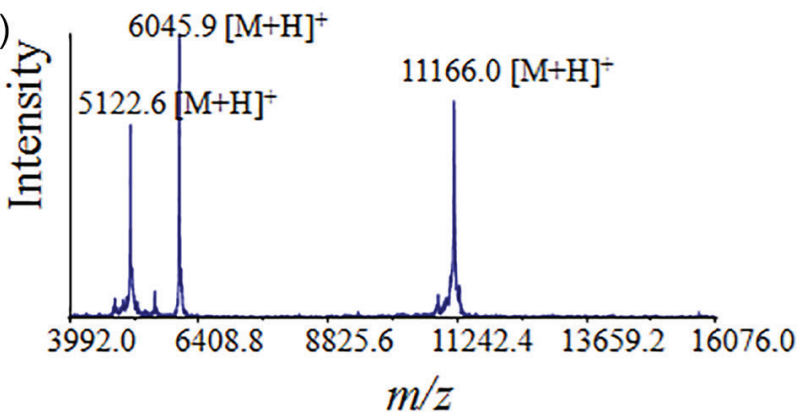

(d)

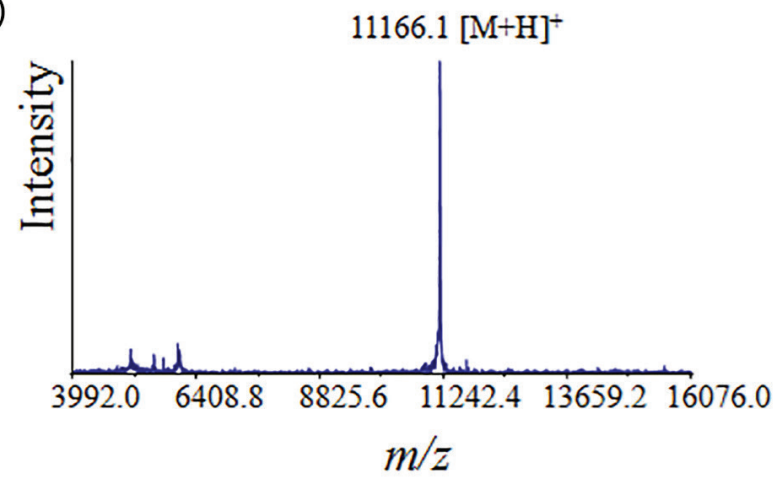

between two GCN-PNA1 molecules or two Max-PNA2 molecules. Even though the disulfide bond formation was not complete in the low concentration of dsPNA duplex (Figures $13 \mathrm{~b}$ and 13c), the monomers stayed in the reduced form without random cross-linking. So, at a very dilute concentration, PNA duplex formation seemed to be the rate-limiting step. Once the two thiols were brought into proximity in the duplex, formation of the disulfide bond by oxidation could readily take place.

This study shows that one can use Watson-Crick base pairing to direct disulfide bond formation. Since the chemistry of PNA synthesis is fully compatible with peptide chemistry and chemical ligation, PNA is much better than DNA to be practically used in templated synthesis. (e)

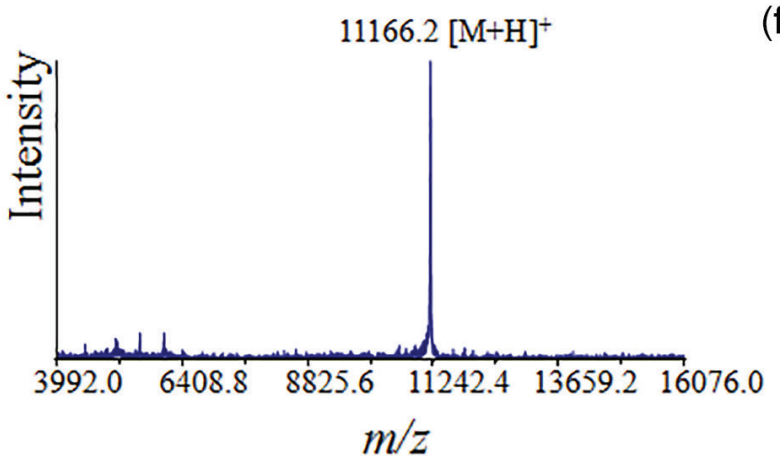

(f)

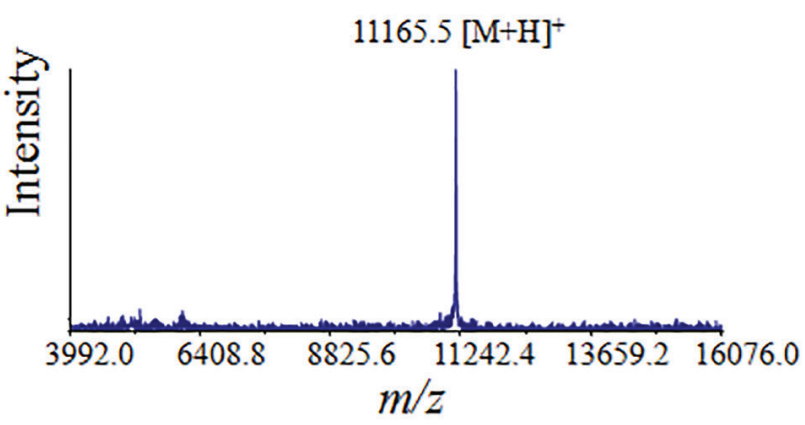

Figure 13. MALDI-TOF mass spectra of oxidation products in different concentration. (a) Before oxidization; (b) $0.5 \mu \mathrm{M}$; (c) $1 \mu \mathrm{M}$; (d) $5 \mu \mathrm{M}$; (e) $10 \mu \mathrm{M}$; (f) $20 \mu \mathrm{M}$. The MW (average) calcd. of GCN-PNA1 is 6044.7, [M + H] $]^{+}$found: 6046.2; the MW calcd. (average) of Max-PNA2 is 5121.5 , [M + H] found: 5122.2; the MW (average) calcd. of GCN-PNA1-S-S-Max-PNA2 is 11164.2, [M + H] found: 11166.2 . 


\section{Conclusions}

In this study, we successfully demonstrated a strategy for specific disulfide bond formation through PNA hybridization. Peptides were linked to a pair of complementary PNAs by native chemical ligation and PNA base pairing brought the two peptides into close proximity for interchain disulfide bond formation. Since PNA directed system is easier to manipulate than DNA system, this system will be more useful and have a broader application scope in the construction of complex biomolecular structures.

\section{Acknowledgments}

This work was kindly supported by Natural Science Foundation of Tianjin City (No. 18JCZDJC34500), State Key Laboratory of Medicinal Chemical Biology (2019003), and Spring Plan of Tianjin First Central Hospital (No. CM201813).

\section{Author Contributions}

Lin Cai did the synthesis of peptides, ligation experiments, data analysis. Wen Hou designed the study, wrote this paper and offered the financial support.

\section{References}

1. Nielsen, P. E.; Egholm, M.; Berg, R. H.; Buchardt, O.; Science 1991, 254, 1497.
2. Watson, J. D.; Crick, F. H. C.; Nature 1953, 171, 737.

3. Hanvey, J. C.; Peffer, N. J.; Bisi, J. E.; Thomson, S. A.; Cadilla, R.; Josey, J. A.; Ricca, D. J.; Hassman, C. F.; Bonham, M. A.; $\mathrm{Au}$, K. G.; Science 1992, 258, 1481.

4. Niemeyer, C. M.; Koehler, J.; Wuerdemann, C.; ChemBiochem 2002, 3, 242.

5. Müller, J.; Niemeyer, C. M.; Biochem. Biophys. Res. Commun. 2008, 377, 62 .

6. Wilner, O. I.; Weizmann, Y.; Gill, R.; Lioubashevski, O.; Freeman, R.; Willner, I.; Nat. Nanotechnol. 2009, 4, 249.

7. Wilner, O. I.; Shimron, S.; Weizmann, Y.; Wang, Z. G.; Willner, I.; Nano Lett. 2009, 9, 2040.

8. Gartner, Z. J.; Liu, D. R.; J. Am. Chem. Soc. 2001, 123, 6961.

9. McKee, M. L.; Milnes, P. J.; Bath, J.; Stulz, E.; Turberfield, A. J.; O'Reilly, R. K.; Angew. Chem., Int. Ed. Engl. 2010, 49, 7948.

10. Gartner, Z. J.; Kanan, M. W.; Liu, D. R.; J. Am. Chem. Soc. 2002, 124, 10304.

11. Snyder, T. M.; Liu, D. R.; Angew. Chem., Int. Ed. Engl. 2005, 44, 7379 .

12. Hannsen, M. H.; Blakskjaer, P.; Petersen, L. K.; Hansen, T. H.; Højfeldt, J. W.; Gothelf, K. V.; Hansen, N. J.; J. Am. Chem. Soc. 2009, 131, 1322.

13. Dose, C.; Seitz, O.; Bioorg. Med. Chem. 2007, 16, 65.

14. Burlina, F.; Dixson, D. D.; Doyle, R. P.; Chassaing, G.; Boddy, C. N.; Dawson, P.; Offer, J.; Chem. Commun. 2008, 2785.

Submitted: August 7, 2019

Published online: November 22, 2019 